NASA Technical Memorandum 102437

AIAA-90-0353

\title{
Buoyancy Effects on the Vapor Condensation Rate on a Horizontal Liquid Surface
}

Mohammad M. Hasan

National Aeronautics and Space Administration

Lewis Research Center

Cleveland, Ohio

and

Chin-Shun Lin

Analex Corporation

NASA Lewis Research Center

Cleveland, Ohio

Prepared for the

28th Aerospace Sciences Meeting

sponsored by the American Institute of Aeronautics and Astronautics

Reno, Nevada, January 8-11, 1990

\section{N/SA}


BUOYANCY EFFECTS ON THE VAPOR CONDENSATION RATE ON A HORIZONTAL LIQUID SURFACE

\author{
Mohammad M. Hasan* \\ National Aeronautics and Space Administration \\ Lewis Research Center \\ Cleveland, Ohio 44135 \\ and \\ Chin-Shun Lin* \\ Analex Corporation \\ NASA Lewis Research Center \\ Cleveland, Ohio 44135
}

\begin{abstract}
SUMMARY
The paper presents the results of a numerical investigation of the effects of buoyancy on the direct condensation of saturated or nearly saturated vapor on a horizontal liquid surface in a cylindrical tank. The liquid motion beneath the liquid-vapor interface is induced by an axisymmetric laminar jet of subcooled liquid. Analysis and numerical results show that the dominant parameter which determines the influence of buoyancy on the condensation rate is the Richardson number. However, the effect of buoyancy on the condensation rate cannot be quantified in terms of Richardson number alone. The critical value of the Richardson number below which the condensation rate is not significantly reduced depends on the Reynolds number as well as the Prandtl number.
\end{abstract}

\title{
NOMENCLATURE
}

A area, jet, outflow or interface

B tank to jet diameter ratio, $D / d$

$C_{p} \quad$ specific heat-at-constant-pressure

D tank diameter

d jet diameter

Gr Grashof number, $g \beta\left(T_{s}-T_{j}\right) D^{3} / v^{2}$

g acceleration due to gravity

$h_{c} \quad$ local condensation heat transfer coefficient, $m_{c} h_{f g} /\left(T_{s}-T_{j}\right)$

$\bar{h}_{c} \quad$ average condensation heat transfer coefficient, $\bar{m}_{c} h_{f g} /\left(T_{s}-T_{j}\right)$

$h_{f g}$ latent heat of condensation

Ja Jakob number, $C_{p}\left(T_{s}-T_{j}\right) / h_{f g}$

*Member, AIAA. 


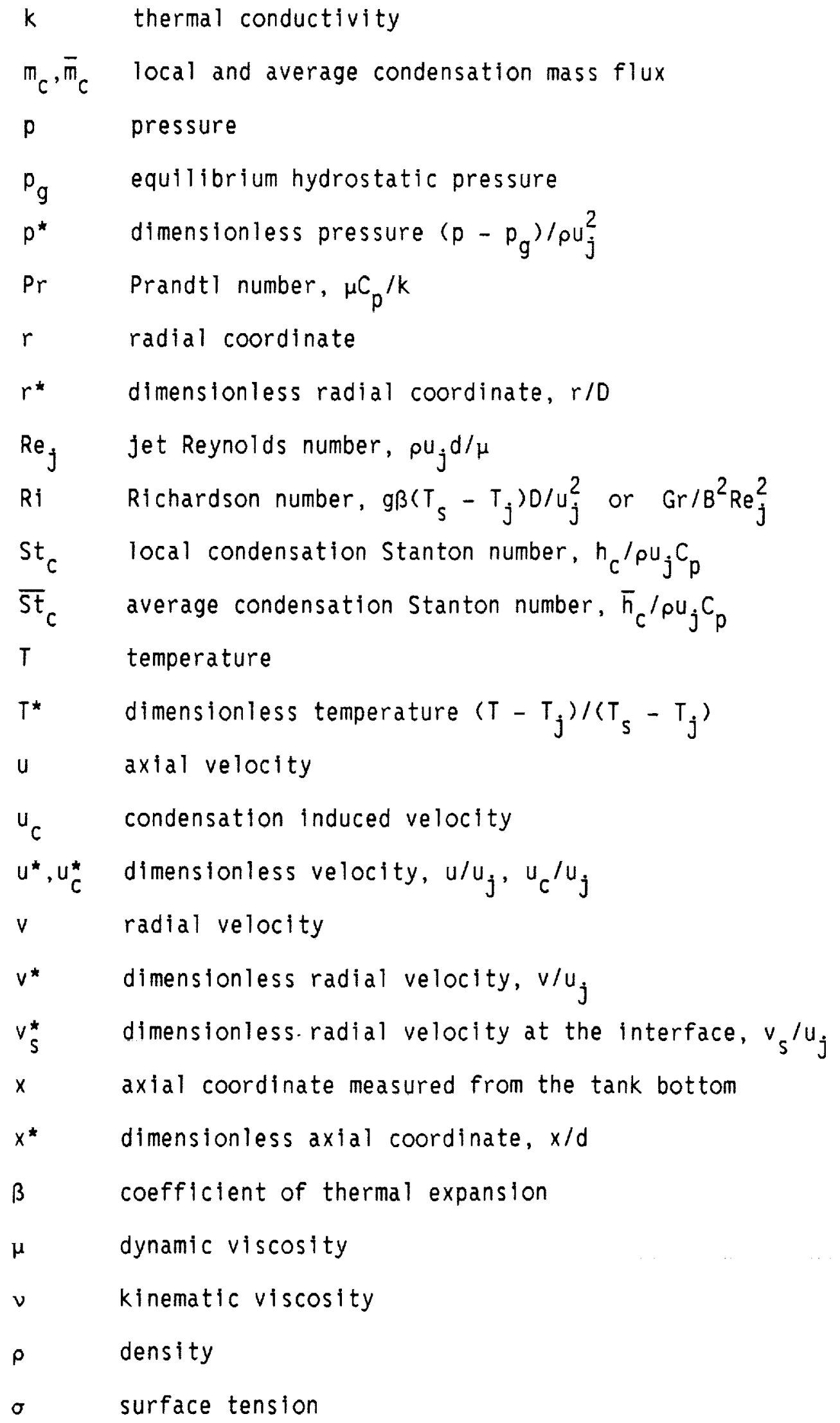




\author{
Subscripts: \\ $j \quad$ evaluated at jet inlet \\ 1 evaluated at liquid condition \\ s evaluated at liquid-vapor interface \\ $v \quad$ evaluated at vapor condition \\ - evaluated at zero Richardson number
}

\title{
INTRODUCTION
}

The paper is concerned with the formulation and numerical computation of the rate of vapor condensation on a liquid surface. The problem under consideration has direct relevance to space-based systems related to cryogenic fluid storage and the control of cryogenic tank pressure. The liquid-vapor interface location and configuration are not well defined in microgravity environments. Therefore, direct venting of vapor to control the tank pressure either during storage or during the filling process of an empty tank is not a viable option. Technology requirements and fluid dynamics and heat transfer problems associated with the cryogenic fluid management in space environments are adequately described in numerous publications (refs. 1 to 4 ). In space systems, the preferred method of storage tank pressure control is to clrculate the liquid through a cooler, reinject the subcooled liquid into the tank and induce vapor condensation at the liquid surface. Therefore, the prediction of the condensation rate in terms of the injection flow conditions and system parameters that may affect the condensation process is required.

The interface condensation process which is principally controlled by the liquid motion beneath the interface is of specific interest. The liquid motion beneath the interface may be induced by an axial jet of subcooled liquid. Recently, Sonin and his coworkers (refs. 5 to 8 ) have performed extensive experimental investigations on the condensation rate of steam on a turbulent liquid surface. The turbulence in the liquid side was produced by a single axial jet directed toward the liquid surface from below. A general empirical correlation for the condensation rate in terms of the liquid-side turbulence properties and fluid properties was developed. The effects of buoyancy on the condensation rate is described in terms of Richardson number. It is shown (refs. 6 and 8 ) for lower jet Reynolds numbers and a Richardson number greater than unity, the condensation rate is significantly reduced even for the turbulent jet. This is due to the thermal stratification near the interface because of buoyancy effects. Realistically, for the ground-based condensation experiment, the buoyancy effects cannot be avoided at low jet velocities. Therefore, it is essential to predict the condensation rate elther analytically or numerically for the cases where the buoyancy effects are significant.

Recently, Gerner and Tien (ref. 9) investigated the interfaclal condensation problem on a horizontal liquid surface by employing a simple axisymmetric model. However, the effects of buoyancy were neglected. Lin (ref. 10) and Lin and Hasan (ref. 11) obtained numerical solutions of the condensation of vapor on a horizontal liquid surface in a cylindrical tank. The liquid motion beneath the interface is induced by an axisymmetric laminar jet of subcooled 
liquid. The effects of the jet Reynolds number, liquid Prandt 1 number, Jakob number as well as system parameters on the condensation rate are investigated. Again, the effect of buoyancy was neglected.

The present paper is an extension of the work reported by Lin (ref. 10). The specific focus of the present work is to investigate the effects of buoyancy on the vapor condensation rate.

\section{PROBLEM FORMULATION}

The physical system and the coordinates used to analyze the problem are shown in figure 1. A cylindrical tank of diameter, $D$, is filled with liquid up to a height $x_{s}$. The liquid surface is in contact with saturated vapor and the tank pressure is kept constant. Therefore, the liquid vapor interface is at a constant saturation temperature corresponding to the tank pressure. An axisymmetric, subcooled laminar jet is introduced at the center of the tank bottom and is directed toward the liquid vapor interface. The liquid is withdrawn from the outer portion of the tank bottom allowing the liquid height to remain constant.

In the present analysis it is assumed that the fluid flow and the temperature fields in the liquid region remain essentially unaffected by the vapor region (refs. 9 and 10). Therefore, the momentum equations and the energy equation in the liquid side can be solved simultaneously and the condensation rate can be determined from the resulting temperature gradient at the interface. The above simplification is based on the following assumptions:

- The vapor is quiescent and at saturation temperature or if superheated, $C_{p}\left(T_{v}-T_{s}\right) / h_{f g} \ll 1$. Therefore, the energy equation in the vapor phase need not be considered.

- The liquid vapor interface is flat and wave free (i.e., $v_{s}^{2} / g D<<1$ and $\left.u_{j} /(g \sigma / \rho)^{1 / 4} \ll 1\right)$.

- The interface is shear free (i.e., $\mu_{v} / \mu_{1} \ll 1$ ).

The governing conservation equations in the nondimensional form can be written as:

$$
\begin{aligned}
& \frac{\partial u^{\star}}{\partial x^{\star}}+\frac{\partial r^{\star} v^{\star}}{r^{\star} \partial r^{\star}}=0 \\
& \frac{\partial u^{\star}}{\partial x^{\star}}+\frac{\partial u^{\star} r^{\star} v^{\star}}{r^{\star} \partial r^{\star}}=-\frac{\partial p^{\star}}{\partial x^{\star}}+\frac{1}{B \operatorname{Re} j}\left[\frac{\partial^{2} u^{\star}}{\partial x^{\star}}+\frac{\partial}{r^{\star} \partial r^{\star}}\left(r^{\star} \frac{\partial u^{\star}}{\partial r^{\star}}\right)\right]+R i T^{\star}
\end{aligned}
$$

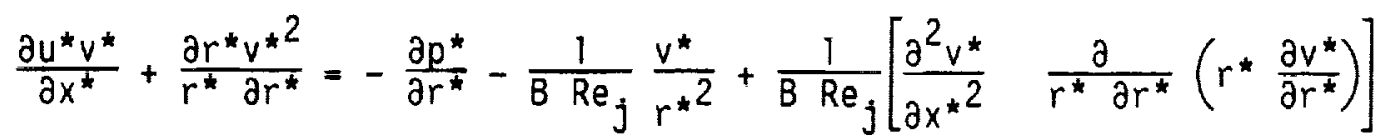




$$
\frac{\partial u^{\star} T^{\star}}{\partial x^{\star}}+\frac{\partial r^{\star} V^{\star} T^{\star}}{r^{\star} \partial r^{\star}}=\frac{1}{B \operatorname{Re}_{j} \operatorname{Pr}}\left[\frac{\partial^{2} T^{\star}}{\partial x^{\star} 2}+\frac{\partial}{r^{\star} \partial r^{\star}}\left(r^{\star} \frac{\partial T^{\star}}{\partial r^{\star}}\right)\right]
$$

The effect of buoyancy is accounted for by retaining the body force term $g \beta\left(T-T_{j}\right)$, (Boussinesq approximation) in the $x$-direction momentum equation, where $g$ is the acceleration due to gravity acting normal to the surface in the negative $x$-direction. In equation (2) the effect of buoyancy is represented by the Richardson number, $R i$.

The following boundary conditions are used to solve elliptic equations (1) to (4). At the centerline, the symmetric conditions are:

$$
v^{\star}=0 \quad \frac{\partial u^{*}}{\partial r^{*}}=\frac{\partial T^{\star}}{\partial r^{\star}}=0
$$

At the solid walls

$$
u^{*}=v^{*}=0
$$

The bottom and side walls are assumed to be adiabatic:

$$
\frac{\partial T^{*}}{\partial x^{\star}}=\frac{\partial T^{\star}}{\partial r^{\star}}=0
$$

At the jet inlet

$$
u^{*}=1 \quad v^{*}=0 \quad T^{*}=0
$$

The axial velocity at the interface is the condensation induced velocity and is given by

$$
u_{C}^{*}=-\frac{J a\left(\frac{\partial T^{*}}{\partial x^{*}}\right)_{s}}{B \operatorname{Pr} \operatorname{Re}_{j}} \quad \text { at } \quad x^{*}=\frac{x_{s}}{D}
$$

At the liquid-withdrawn plane

$$
\begin{gathered}
u^{*}=-\frac{A_{j}}{A_{\text {out }}}\left[1+\frac{J a\left(\frac{\partial T^{\star}}{\partial x^{*}}\right)_{s} B}{\operatorname{Pr} \operatorname{Re}_{j}}\right] \\
v^{*}=0 \quad \frac{\partial T^{*}}{\partial x^{*}}=0
\end{gathered}
$$

The primary parameters of physical interest are: condensation mass flux

$$
m_{c}=k \frac{\left(\frac{\partial T}{\partial x}\right)_{s}}{h_{f g}}
$$


and the local and average condensation heat transfer coefficients and can be written in terms of stanton numbers as:

$$
\begin{gathered}
S t_{c}=\frac{h_{c}}{\rho u_{j} C_{p}}=\frac{\left(\frac{\partial T^{*}}{\partial x^{*}}\right)_{s}}{B \operatorname{Pr} \operatorname{Re}_{j}} \\
\overline{s t}_{c}=\frac{\bar{h}_{c}}{\rho u_{j} C_{p}}=\frac{8 \int_{0}^{0.5}\left(\frac{\partial T^{\star}}{\partial x^{*}}\right)_{s} r^{\star} d r^{*}}{B \operatorname{Pr} \operatorname{Re}_{j}}
\end{gathered}
$$

The nondimensional form of governing equations and the boundary conditions show that the average condensation stanton number can be expressed as:

$$
\overline{s t}_{c}=f\left(\operatorname{Re}_{j}, R i, \operatorname{Pr}, J a, \frac{d}{D}, \frac{X_{s}}{D} \text {, and } \frac{A_{\text {out }}}{A_{j}}\right)
$$

The effects of system parameters, Ja and $\mathrm{Pr}$ have been investigated in references 10 and 11. The focus of this study is to investigate the effects of buoyancy, described by the Richardson number, on the condensation process.

\section{NUMERICAL SOLUTION}

The coupled elliptic partial differential equations along with the boundary conditions are solved by a finite difference method. Calculations are performed with a nonuniform grid distribution with the concentration of grid nodes in the centerline, near wall and near interface regions where the gradients of the flow properties are expected to be large. The essential features of the numerical scheme are described in references 10 and 11 . Reference 10 shows that $72 \times 41$ grid nodes gives reasonably grid-independent solutions. The same grid distribution is used in the present computation. It should be noted that the coupling of momentum and energy equations is due to the buoyancy term as well as the condensation induced velocity, $u_{c}$, at the interface. However, numerical results (ref. 11) show that the temperature and velocity fields in the liquid side, including the near-interface region, remain essentially unaffected by the condensation induced velocity, $u_{C}$, if $\mathrm{Ja}<0.2$. Therefore, in the present calculation the condensation induced velocity is set equal to zero. The implication is that the results presented in this paper and conclusions drawn are valid only for Ja $<1$.

\section{RESULTS AND DISCUSSION}

Numerical calculations are performed for fixed values of the geometric parameters, $D / d=20$ and $x_{s} / D=1$ for the jet Reynolds number ranging from 150 to 450 and Prandtl numbers of 1.25 and 2.65 . The value of the Richardson number is varied from 0 to 0.2 . 
In the analysis, the buoyancy effect is accounted for in the Boussinesq approximation. The nondimensional form of the equations show that the Richardson number scales the buoyancy effects. In a gravitational field, buoyancy creates a warm thermal layer near the interface which reduces the condensation rate. Therefore, the velocity and temperature distributions near the interface and the interface temperature gradient are of interest for the purpose of analysis. Some representative velocity and interface temperature gradient distributions are presented in the paper. Figures 2 and 3 show the radial distribution of axial velocity at two locations near the interface for the jet Reynolds number of 300 and Prandtl number of 1.25 . The results show that as the Richardson number increases the jet velocity in the jet influenced region decreases. This occurs because the jet fluid temperature is lower than the surrounding fluid temperature and, therefore, in this case buoyancy opposes the jet flow. Figure 3 shows that for $R i=0.2$, flow reversal occurs near the outer part of the jet influence region. The effects of the Richardson number on the radial velocity at the free surface $\left(x^{*}=1\right)$ and near the free surface $\left(x^{*}=0.97\right)$ are shown in figures 4 and 5 . The results show that the radial outflow velocity is significantly reduced with the increase of Richardson number. A reduction in the radial outflow velocity near the interface reduces the rate at which the heat of condensation can be transported away from the interface.

For a fixed Reynolds number the radial distribution of the temperature gradient at the interface for different values of the Richardson number is shown in figure 6 . The average value of the condensation heat flux is obtained by integrating this profile over the entire interface area. The results are shown in figures 7 and 8 for $\operatorname{Pr}=1.25$ and 2.65 , respectively, where the ratio of the average Stanton number to stanton number for zero Richardson number, $S t_{c} / S t_{c o}$ is plotted as a function of Richardson number. It can be observed from figures 7 and 8 that the condensation rate decreases significantly with increase in Richardson number. Also, the effect of buoyancy on the condensation rate cannot be quantified in terms of the Richardson number alone. The critical value of the Richardson number below which the stanton number is not significantly reduced depends on Reynolds number as well as Prandtl number. However, analyses of the results show that for $\mathrm{Ri}<0.1$, the effect of buoyancy on the vapor condensation rate can be neglected for the range of Reynolds numbers and Prandt 1 numbers covered in the present investigation.

\section{CONCLUSION}

The effects of buoyancy on the direct condensation of vapor on a horizontal liquid surface in a cylindrical tank are investigated numerically. The liquid motion beneath the interface is induced by an axisymmetric laminar jet of subcooled liquid. It is shown that the Richardson number scales the buoyancy effects. For fixed values of the geometric parameters, $D / d=20$ and $x_{s} / D=1$, numerical solutions are obtained for Richardson number varying from 0 to 0.2 .

The results show that the condensation rate is significantly reduced for higher values of the Richardson number. However, the reduction of the condensation rate cannot be quantified in terms of Richardson number alone since it also depends on both the jet Reynolds number and the Prandtl number. 


\section{REFERENCES}

1. Aydelott, J.C., Carney, M.J., and Hochstein, J.I., "NASA Lewis Research Center Low-Gravity Fluid Management Technology Program," Man's Permanent Presence in Space, AIAA, New York, 1985 (NASA TM-87145).

2. Aydelott, J.C. and Rudland, R.S., "Technology Requirements to be Addressed by NASA Lewis Research Center Cryogenic Fluid Management Facility Program," AIAA Paper 85-1229, July 1985 (NASA TM-87048).

3. Poth, L.J. Jr. and Van Hook, J.R., "Control of the Thermodynamic State of Space-Stored Cryogens by Jet-Mixing," Journal of Spacecraft and Rockets, Vol. 9, No. 5, May 1972, pp. 332-336.

4. Chato, D.J., "Thermodynamic Modeling of the No-Vent Fill Methodology for Transferring Cryogens in Low-Gravity," AIAA Paper 88-3403, July 1988 (NASA TM-100932).

5. Sonin, A.A., Shimko, M.A., and Chun, J.H., "Vapor Condensation Onto a Turbulent Liquid. 1. The Steady State Condensation Rate as a Function of Liquid-Side Turbulence," International Journal of Heat and Mass Transfer, Vol. 29, No. 9, 1986, pp. 1319-1322.

6. Helmick, M.R., Khoo, B.C., Brown, J.S., and Sonin, A.A., "Vapor Condensation Rate at a Turbulent Liquid Interface for Application to Cryogenic Hydrogen," AIAA Paper 88-0559, Jan. 1988.

7. Brown, J.S., Helmick, M.R., and Sonin, A.A., "Vapor Condensation at a Turbulent-Liquid Surface in Systems with Possible Space-Based Applications," AIAA Paper 89-2846, 1989.

8. Brown, J.S., Khoo, B.C., and Sonin, A.A., "Rate Correlation for Condensation of Pure Vapor on Turbulent, Subcooled Liquid," International Journal of Heat and Mass Transfer, 1989, to be published.

9. Gerner, F.M. and Tien, C.L., "Axisymmetric Interfacial Condensation Mode1," Journal of Heat Transfer, Vol. 111, No. 2, May 1989, pp. 503-510.

10. Lin, C.S., "Numerical Studies of the Effects of Jet-Induced Mixing on Liquid-Vapor Interface Condensation," AIAA Paper 89-1744, June 1989.

11. Lin, C.S. and Hasan, M.M., "Vapor Condensation on Liquid Surface Due to Laminar Jet-Induced Mixing: The Effects of System Parameters," AIAA Paper 90-0354, Jan. 1990 (NASA TM-102433). 


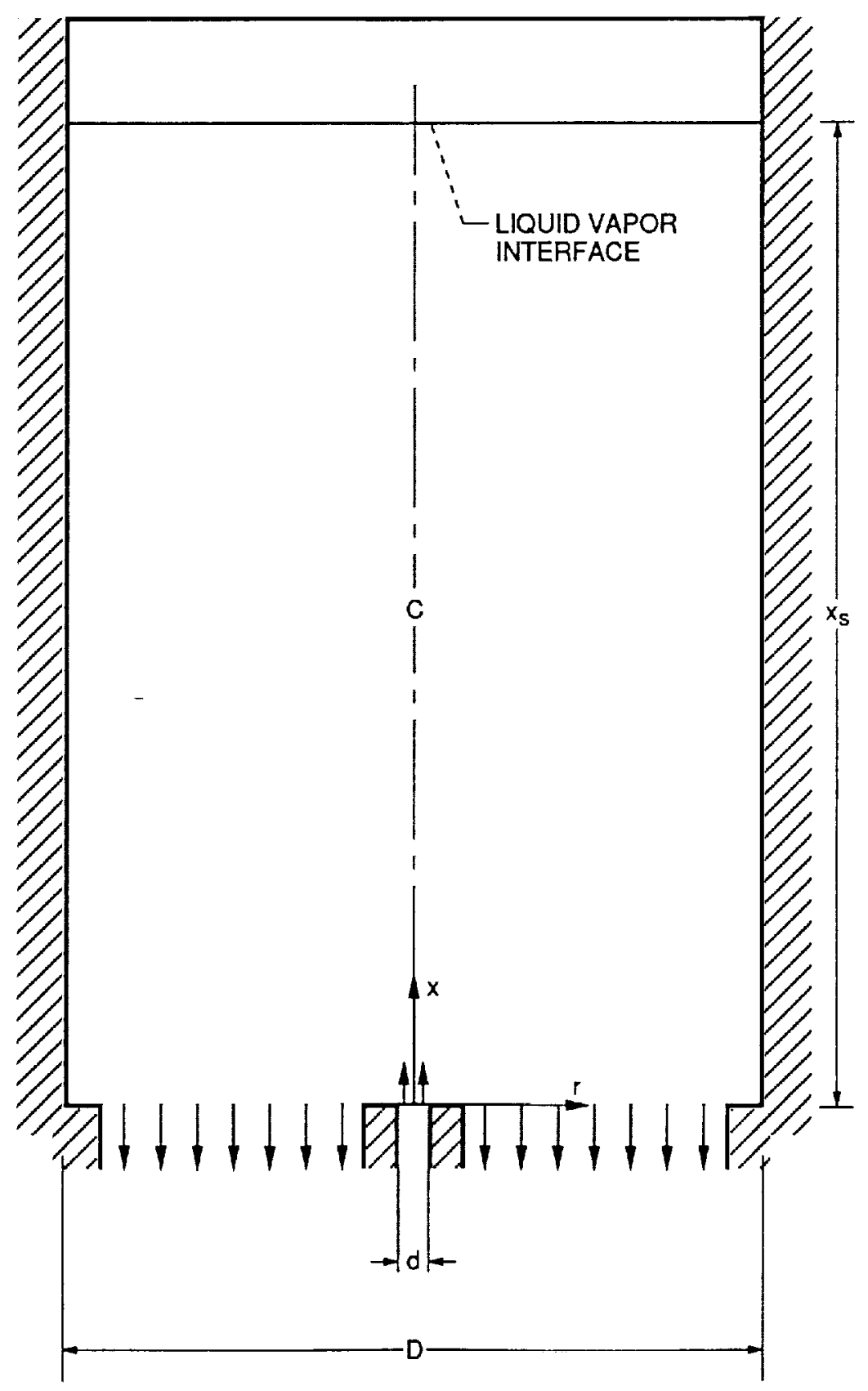

Figure 1. - Physical system and coordinates. 


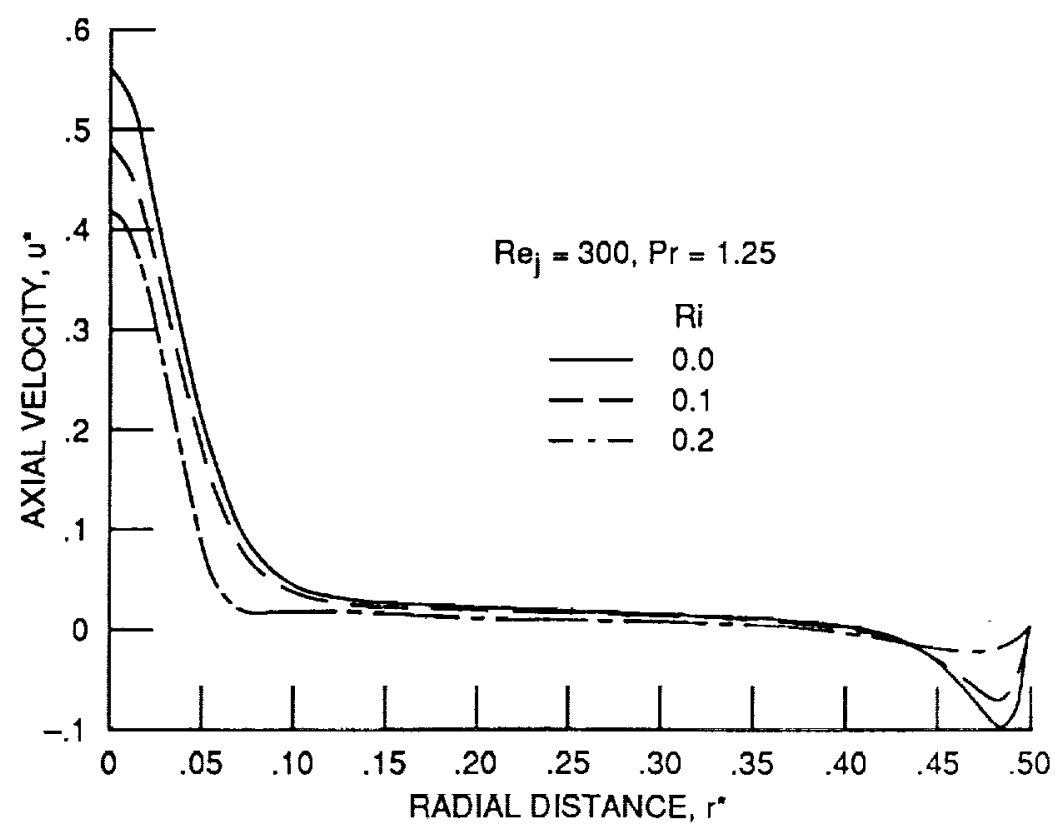

Figure 2. - Effect of Richardson number on the radial distribution of axial velocity, $x^{*}=.97$.

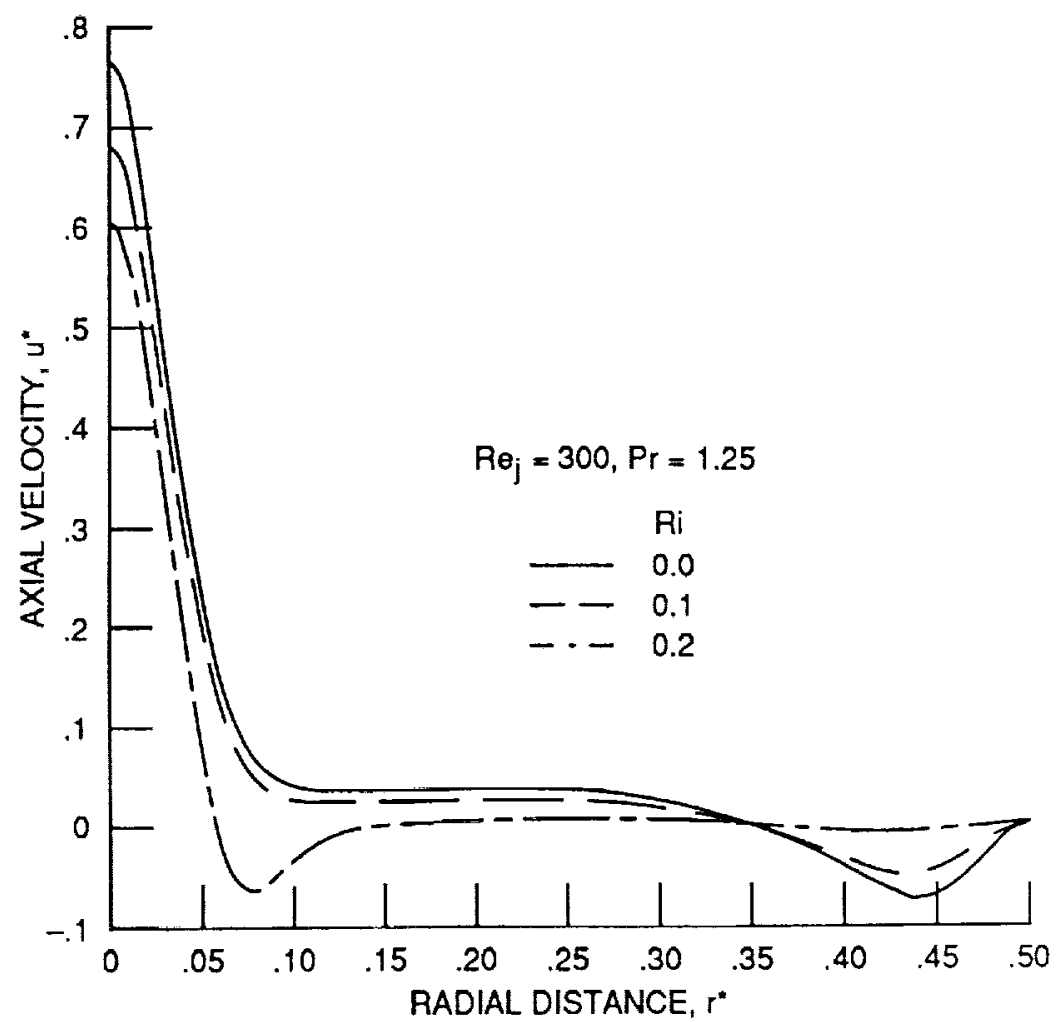

Figure 3. - Effect of Richardson number on the radial distribution of axial velocity, $x^{*}=.81$. 


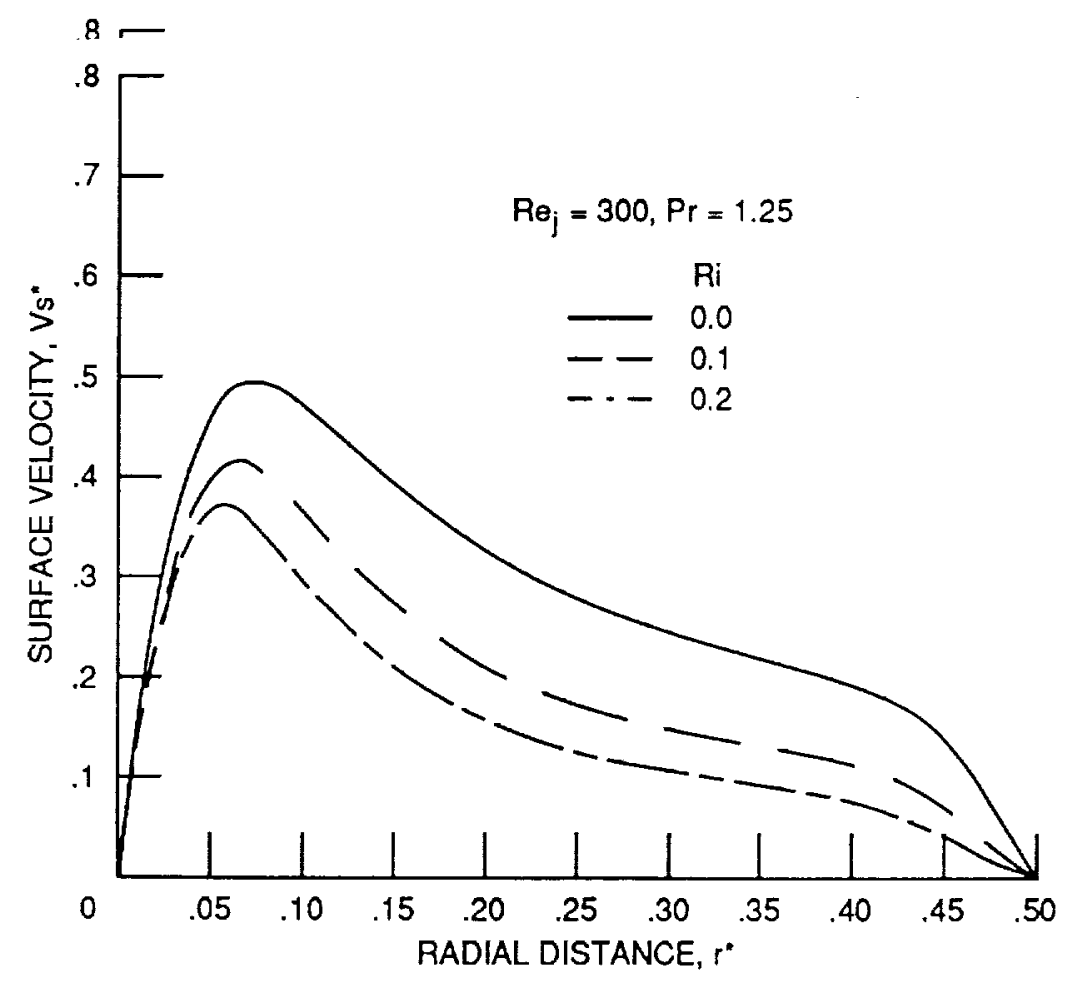

Figure 4. - Effect of Richardson number on the radial velocity at the free surface.

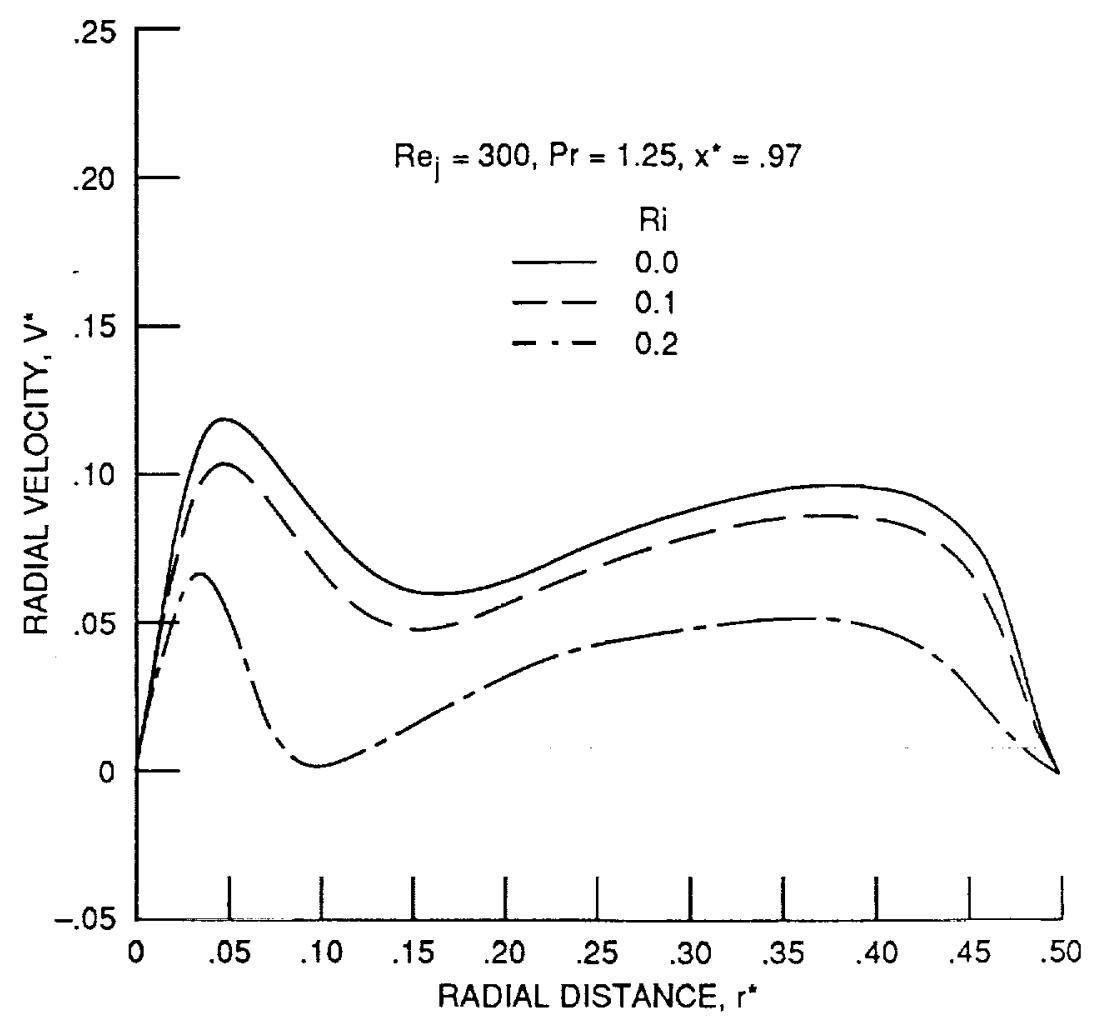

Figure 5. - Effect of Richardson number on the radial velocity near the interface. 


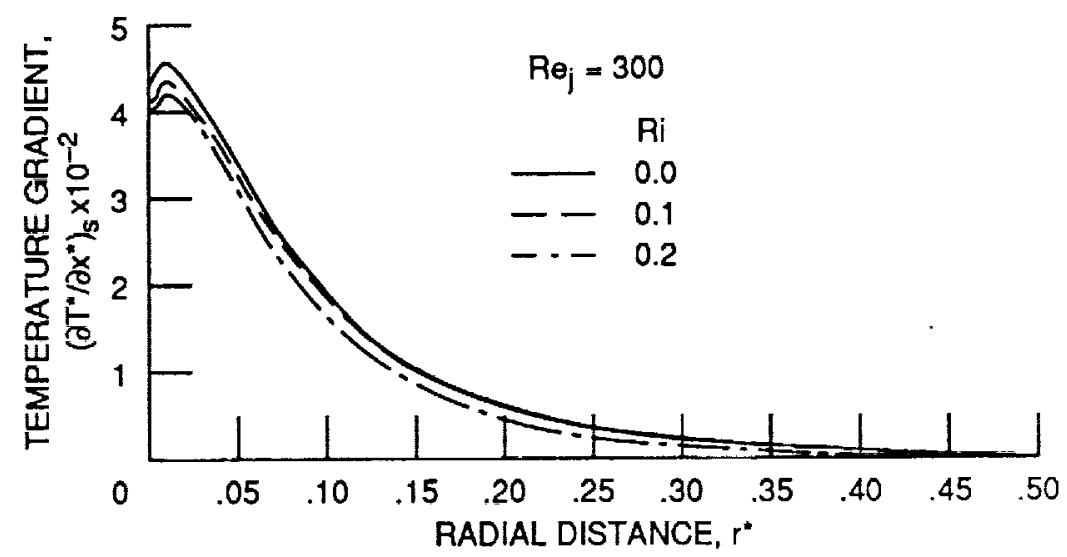

Figure 6. - Elfect of Richardson number on the temperature gradient profiles at the interface.

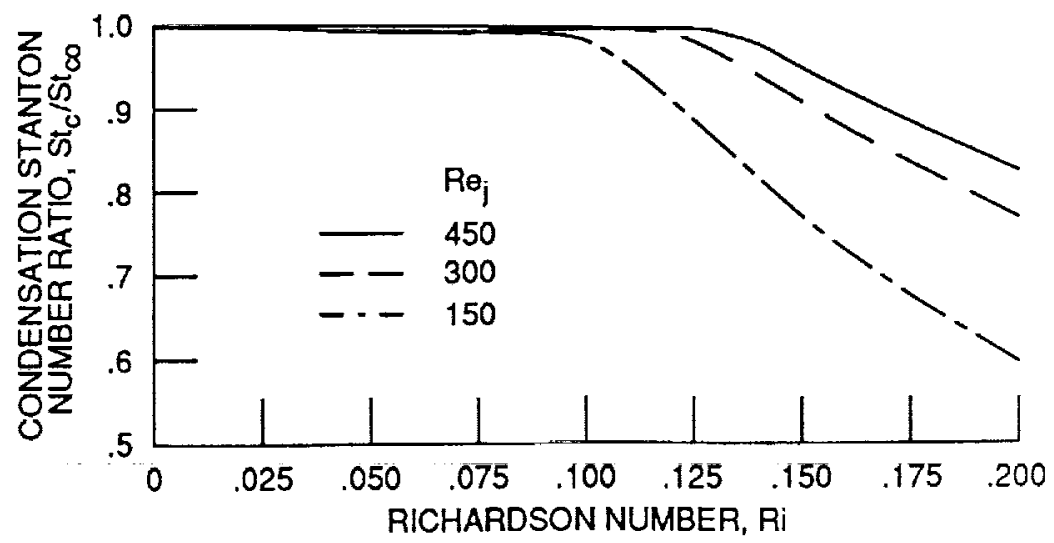

Figure 7. - Average condensation Stanton number ratio as a function of Richardson number for $\mathrm{Pr}=1.25$.

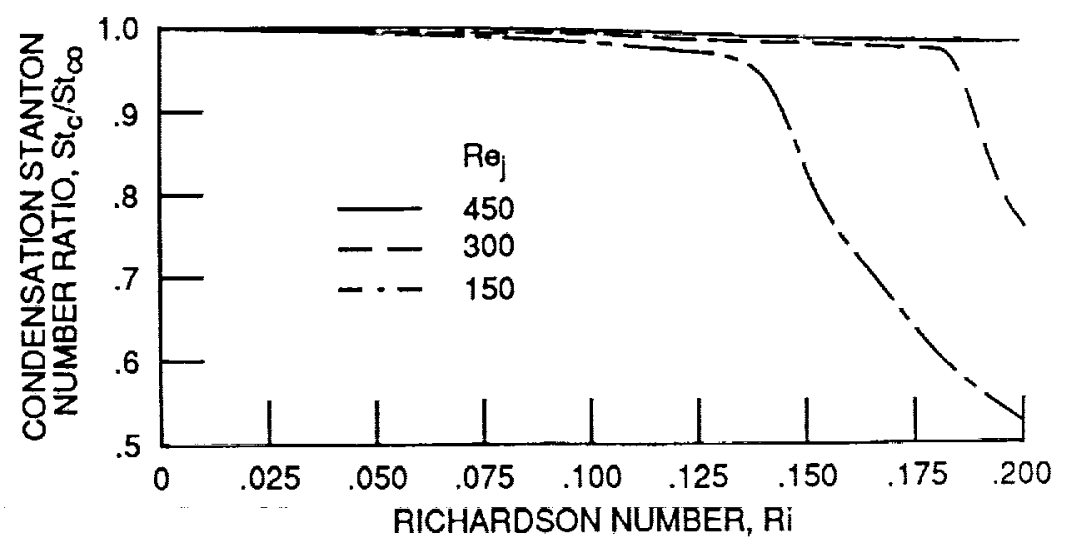

Figure 8. - Average condensation Stanton number ratio as a function of Richardson number for $\mathrm{Pr}=2.65$. 


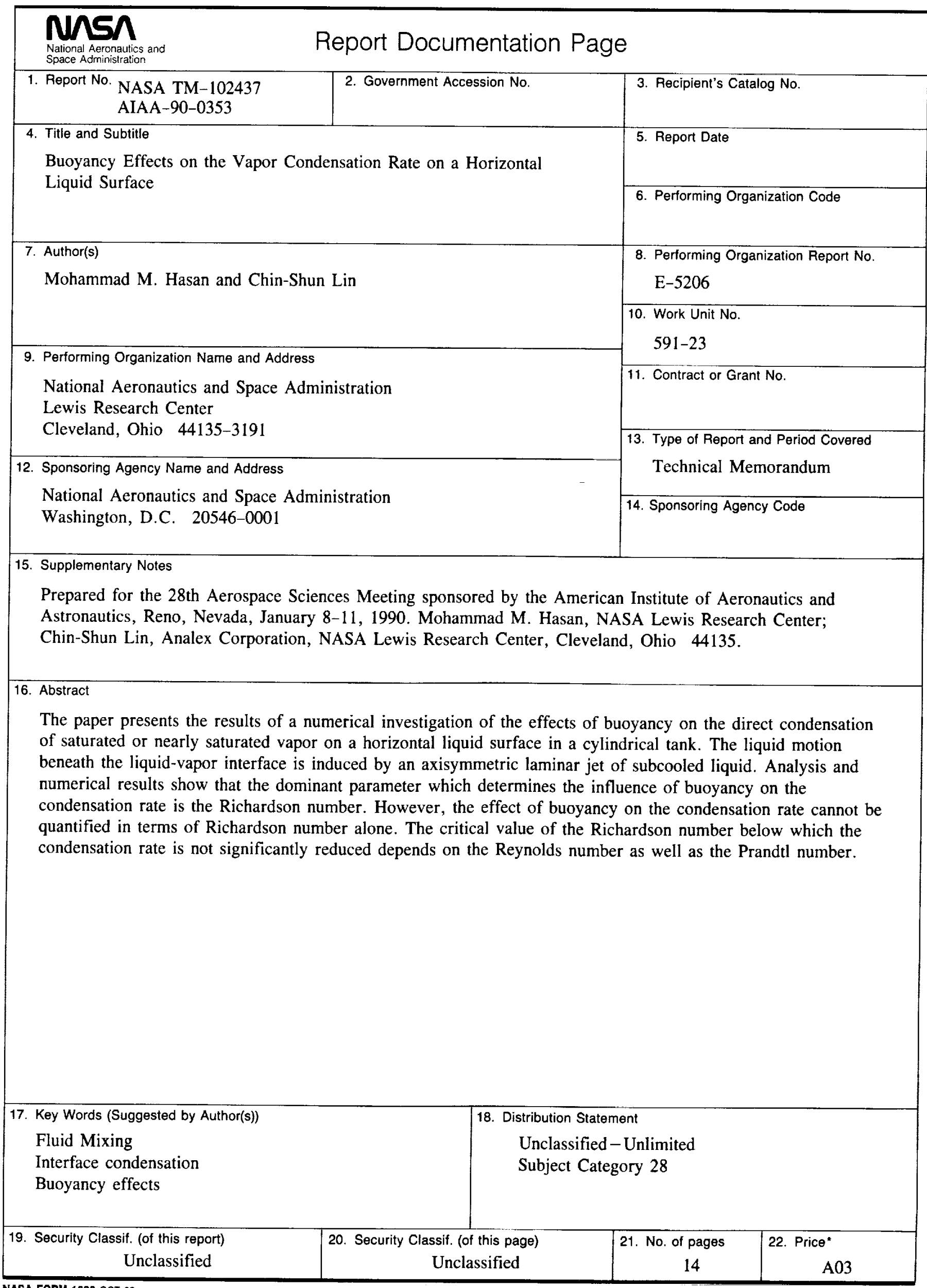


